\title{
Evolution des caractéristiques de la graine et du capitule chez le tournesol au cours de la maturation
}

\author{
B. Chervet et F. Vear
}

INRA, Station d'amélioration des plantes, Domaine de Crouelle, 63039 Clermont-Ferrand Cedex, France

(reçu le 13-6-1988, accepté le 19-12-1988)

\begin{abstract}
Résumé - L'évolution de l'humidité du capitule et des graines de 25 variétés de tournesol (Helianthus annuus L.), de la teneur en huile de 13 d'entre elles et du poids sec de 100 grains, pour 10 d'entre elles, ont été suivis de la floraison jusqu'à la maturité complète. L'humidité des graines diminue régulièrement au cours des 7 semaines d'étude à des vitesses différentes suivant les variétés. L'humidité du capitule ne varie pas pendant les 6 premières semaines après la floraison (70 à $80 \%$ d'eau), puis décroît de façon plus ou moins importante suivant les variétés. Un certain nombre de corrélations entre différents caractères et l'humidité au 2 septembre sont établies. Le contenu maximal en huile varie, suivant les variétés, de 44 à $52 \%$ du poids sec du grain. Il est élaboré, en moyenne, dans les 40 premiers jours après la floraison, la matière sèche du grain l'étant dans les 45 premiers jours après la floraison. Les vitesses d'évolution des teneurs en huile et en matière sèche varient en fonction des génotypes.
\end{abstract}

dessèchement - matière sèche - teneur en huile - maturité physiologique

Summary - Changes in characteristics of sunflower seed and capitula during ripening. Changes in the humidity of capitula and seed of 25 sunflower (Helianthus annuus L.) varieties were followed from flowering to maturity, the oil content evolution was studied for 13 varieties and the 100 grain weight evolution for 10 . The water content of seeds diminished regularly during the 7 weeks of the study, with significant varietal rate differences. The capitulum humidity did not vary during the six first weeks after flowering (70 to $80 \%$ ) but then was reduced to a variable extent according to variety. Correlation between different characters and humidity on 02/09/87 were established. The maximum oil content of the seed varied between varieties from 44 to $52 \%$ of dry weight. It was generaly produced during the 40 days after flowering, comparable with the dry weight, maximum 45 days after flowering days. The rates of increase of both these characteristics varied according to genotype.

drying - dry matter - oil content - physiological maturity

\section{Introduction}

Chez le tournesol, les principales caractéristiques conduisant à la maturité sont : la diminution de la teneur en eau des graines et du capitule, l'élaboration de la teneur en huile et l'acquisition du poids de 1000 grains. Cette phase de maturation peut être divisée en 2 étapes. L'une va de la floraison à la maturité physiologique, définie par Harrington (1972) comme le maximum de poids sec du grain. L'autre va de la maturité physiologique à la récolte, qui intervient généralement aux alentours de $10 \%$ d'humidité du grain. En France, peu de travaux ont été réalisés sur la maturation chez le tournesol, qui est pourtant d'un intérêt capital pour la prévision de la récolte avec un rendement de bonne qualité en grain et en huile. En 1976, Rollier et al. ont effectué une étude de l'évolution de l'humidité et de la matière sèche des graines avec des variétés inscrites au catalogue officiel à cette époque, mais aucune n'est actuellement cultivée. D'autres travaux analogues ont été réalisés sur différentes variétés par des auteurs américains (Anderson, 1975; Browne, 1978; Robertson et al., 1978; Goyne et al., 1979; Robinson, 1983).

Le but de l'étude entreprise à Clermont-Ferrand était de connaître les différentes caractéris- 
tiques de l'akène et du capitule (teneur en eau, accumulation de matière sèche, teneur en huile de la graine) au cours de la maturation, pour un grand nombre de variétés commercialisées représentant une large gamme de précocité. Nous avons aussi voulu préciser les caractéristiques du stade de maturité physiologique. Cette étape est en effet importante puisqu'elle constitue la fin de la vie active de la plante et peut déterminer la date éventuelle de défoliation ou la disparition des résistances aux pathogènes.

\section{Matériel et Méthodes}

Le dispositif expérimental utilisé était un système bloc avec une seule répétition et 25 variétés commercialisées (Aphasol, Vitaflor, Isomax, 293 D (Pinto), IBH 166, Primasol, Sunking, Frankasol, Rodéo, Prestiflor, DKS 39, Mirasol, Viki, Cargisol, Sirio, Cerflor, Maryflor, Siou, Mikaflor, Véraflor, IS7111, Elia, Pariou, Flamme, Sémira). Chaque parcelle était constituée de 4 lignes de 30 mètres avec un écartement de $60 \mathrm{~cm}$ entre 2 lignes; seules les 2 lignes centrales ont été utilisées pour les prélèvements. II n'y a eu aucune irrigation sur les parcelles.

La date de $50 \%$ de floraison de chaque parcelle a été notée. Afin de suivre l'évolution de la maturation des graines et des capitules, nous avons effectué des prélèvements chaque semaine pendant 7 semaines, les premiers étant réalisés environ 20 jours après la floraison, les derniers correspondant à un stade de récolte. Trois prélèvements par capitule ont été effectués, avec un emporte-pièce de $2,5 \mathrm{~cm}$ de diamètre, sur 4 plantes par variété. Ils ont été faits suivant un angle de $120^{\circ}$ sur les capitules. On avait ainsi 3 échantillons de 20 à 30 graines des rangs du milieu du capitule. Les plantes ont été choisies au hasard lors des premiers prélèvements, puis de façon à être représentatives de la parcelle. Le poids frais et le poids sec, après étuvage pendant $48 \mathrm{~h}$ à $80^{\circ} \mathrm{C}$, des graines et du capitule ont été déterminés. Le poids sec de 100 graines a été déterminé pour 10 variétés. La teneur en huile des graines sèches a été mesurée, pour 13 variétés, par résonance magnétique nucléaire (Gradlung et Zimmerman, 1975). Pour l'humidité, nous avons fait la moyenne des 3 échantillons de chaque capitule, pour les 2 autres paramètres nous avons regroupé les échantillons. L'humidité est calculée de 2 façons. D'une part sur le poids frais de l'ensemble de la graine (eau+huile+fraction protéique+coque) pour les 25 variétés, d'autre part sur la fraction non lipidique de la graine pour les 10 variétés pour lesquelles on dispose du poids de 100 graines.

\section{Résultats}

\section{Evolution du poids sec de 100 graines}

L'évolution du poids sec de 100 graines au cours du temps pour 4 variétés est représentée par la Figure 1. L'allure des courbes varie suivant les génotypes. Dans certains cas, on constate une augmentation à partir du début des mesures jusqu'à un maximum, ensuite la courbe diminue. Frankasol (Fig. 1a), Alphasol (Fig. 1b) et IBH 166 présentent ce type de comportement. Dans d'autres cas, on obtient un palier qui se maintient lors des dernières mesures. Ceci est observé pour Mirasol (Fig. 1a), Pinto (Fig. 1b), Véraflor, Rodéo, et Viki. La quantité de matière sèche au début des mesures (environ 20 jours après la floraison) varie suivant les variétés (Tableau I). Par exemple Mirasol a formé 1,86 gramme de matière sèche pour 100 graines, IBH 1662,21 , Sémira 2,83 , Pinto 1,32. Cependant, chez Mirasol et Sémira, cela représente $55 \%$ du poids sec final de 100 grains et, respectivement, 38 et $30 \%$ pour IBH 166 et Pinto. On observe une corrélation hautement significative $(r=0,70,8 \mathrm{ddl})$ entre la taille de la graine à ce stade et la taille finale de la graine à maturité. La vitesse d'accumulation de matière sèche dans la graine (Tableau I) varie de $0,09 \mathrm{~g} / 100$ graines/jour pour Mirasol à 0,220 pour IBH 166. A partir du $21 \mathrm{e}$ jour après la floraison, la vitesse maximale se maintient pendant 2 semaines chez Frankasol et Pariou, 3 semaines chez Mirasol, Rodéo, Pinto, IBH 166, Véraflor, Sémira et Viki, 4 semaines chez Alphasol. II ne

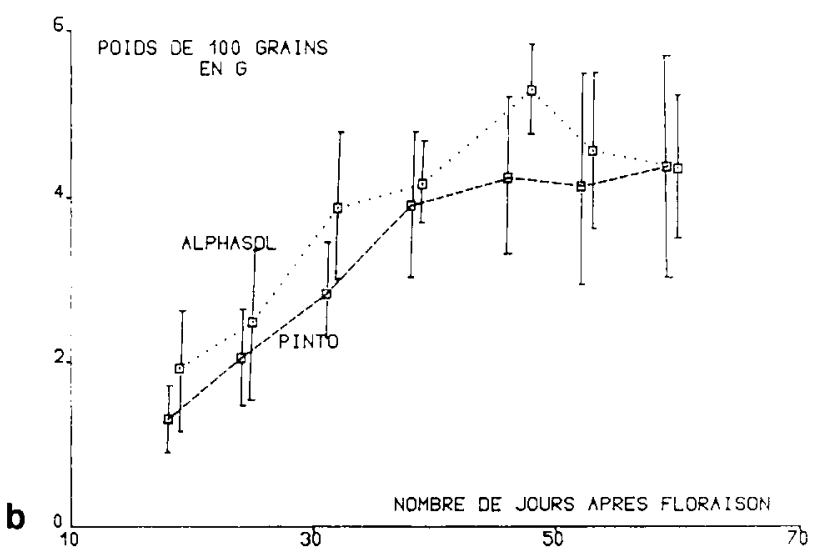

Fig. 1 a et b. Evolution de la matière sèche de 100 graines au cours du temps (intervalle de confiance pour un coefficient de sécurité de $95 \%$ ). 
Tableau I. Valeurs des paramètres étudiés relatifs au poids sec des grains.

$\begin{array}{llll}\text { Poids de } & \text { Nb.j. après } & \text { Humidité } & \text { Vitesse maximale } \\ 100 \text { graines } & \text { floraison } & \text { au maximum } & \text { de remplissage } \\ \text { en g, 20j. } & \text { pour max. } & \text { de poids } & \text { en gde } \\ \text { après floraison } & \text { poids sec } & \text { sec } & \text { MS/100 graines/j }\end{array}$

Poids de 100 graines

à la dernière

observation

Moyenne

groupes homogènes

(Newmann-Keuls à 5\%)

\begin{tabular}{lllllll} 
IBH 166 & 2,21 & 42 & 34 & 0,220 & 5,75 & $\mathbf{a}$ \\
Pariou & 2,55 & 52 & 28 & 0,180 & 5,38 & $\mathrm{ab}$ \\
Sémira & 2,83 & 44 & 35 & 0,123 & 5,18 & $\mathrm{ab}$ \\
Pinto & 1,32 & 46 & 35 & 0,128 & 4,37 & $\mathrm{bc}$ \\
Frankasol & 2,23 & 44 & 30 & 0,180 & 4,36 & $\mathrm{bc}$ \\
Alphasol & 1,92 & 48 & 18 & 0,119 & 4,34 & $\mathrm{bc}$ \\
Rodéo & 1,55 & 44 & 28 & 0,120 & 3,73 & $\mathrm{c}$ \\
Viki & 1,94 & 41 & 33 & 0,100 & 3,69 & $\mathrm{c}$ \\
Mirasol & 1,86 & 43 & 36,5 & 0,090 & 3,42 & $\mathrm{c}$ \\
Véraflor & 1,42 & 43 & 41 & 0,100 & 3,33 & $\mathrm{c}$ \\
\hline
\end{tabular}

** : La vitesse maximale de remplissage est calculée sur la période de croissance linéaire

semble pas y avoir de relation entre la durée et la vitesse de remplissage.

Le maximum du poids sec de 100 graines est atteint au plus tôt 42 jours après floraison, chez Frankasol, au plus tard 52 jours après floraison, chez Alphasol, et la moyenne des 10 variétés est de 45 jours (Tableau I). La terieur en eau du grain pour laquelle le maximum de poids sec est atteint varie de $18 \%$ pour Alphasol à $41 \%$ pour Véraflor, et la moyenne pour les 10 variété est de $32 \%$.

Le Tableau I donne le classement des variétés, à la dernière mesure, pour le poids de 100 graines. IBH 166 a les graines les plus grosses, 5,75 grammes, Véraflor les plus petites, 3,33 grammes. On note une corrélation hautement significative $(r=0,82,8 \mathrm{ddl})$ entre le poids de 100 graines et leur vitesse de remplissage.

\section{Evolution de la teneur en huile}

Le Tableau II présente les différents paramètres relatifs à la teneur en huile. Les modalités d'établissement de cette teneur varient en fonction des variétés (Fig. 2). On observe des différences dans la proportion d'huile au début des mesures. Vitaflor a déjà une teneur en huile de $30 \%$ du poids sec 21 jours après floraison, alors que Véraflor est à 10\% (Tableau II).

La teneur en huile augmente plus ou moins rapidement pour atteindre un palier qui varie en fonction des variétés. Alphasol et Pinto atteignent le palier brusquement (Fig. 2a). Pour Pariou (Fig. 2b), il y a un ralentissement avant d'atteindre le palier. Ce dernier est atteint au plus tôt
32 jours après floraison, pour Alphasol, au plus tard 49 jours après floraison, pour Véraflor (Tableau II). La moyenne des 13 variétés est de 40 jours après floraison. Le maximum d'huile est atteint pour $33 \%$ de l'humidité de la graine chez Viki, $50 \%$ chez Alphasol et $40 \%$ pour la moyenne des variétés (Tableau II).

La vitesse maximale d'évolution de la teneur en huile est atteinte par Sunking, qui forme 3,2 points d'huile par jour entre le $21^{\mathrm{e}}$ et le $27^{\mathrm{e}}$ jour après floraison, ensuite par Viki avec 2,9 points d'huile entre le $19 \mathrm{e}$ et le $26 \mathrm{e}$. IS7111 a la vitesse d'évolution la plus faible, 1,3 point d'huile par jour entre le $21^{\mathrm{e}}$ et le $40^{\mathrm{e}}$ jours après floraison.

Parmi les variétés étudiées, la teneur maximale en huile varie de $52,1 \%$ chez Viki à $44,4 \%$ chez Rodéo (Tableau II). Cependant, la différence de 4,3 points d'huile entre Viki et Alphasol n'est pas significative.

\section{Dessèchement des graines et du capitule}

Les Figures 3a, 3b, 3c, 3d montrent les courbes de dessèchement des capitules (1), des graines entières (2), et de la fraction non lipidique des graines (3).

Nous avons comparé l'évolution du pourcentage d'humidité dans la graine entière et dans la fraction non lipidique, afin de déterminer si une partie du dessèchement observé pouvait être expliquée par l'augmentation de l'huile, composé hydrophobe. L'allure des courbes étant sensiblement la même, la diminution d'humidité est donc bien réelle. Ainsi, dans la suite des résultats, nous utiliserons les humidités calculées avec le 

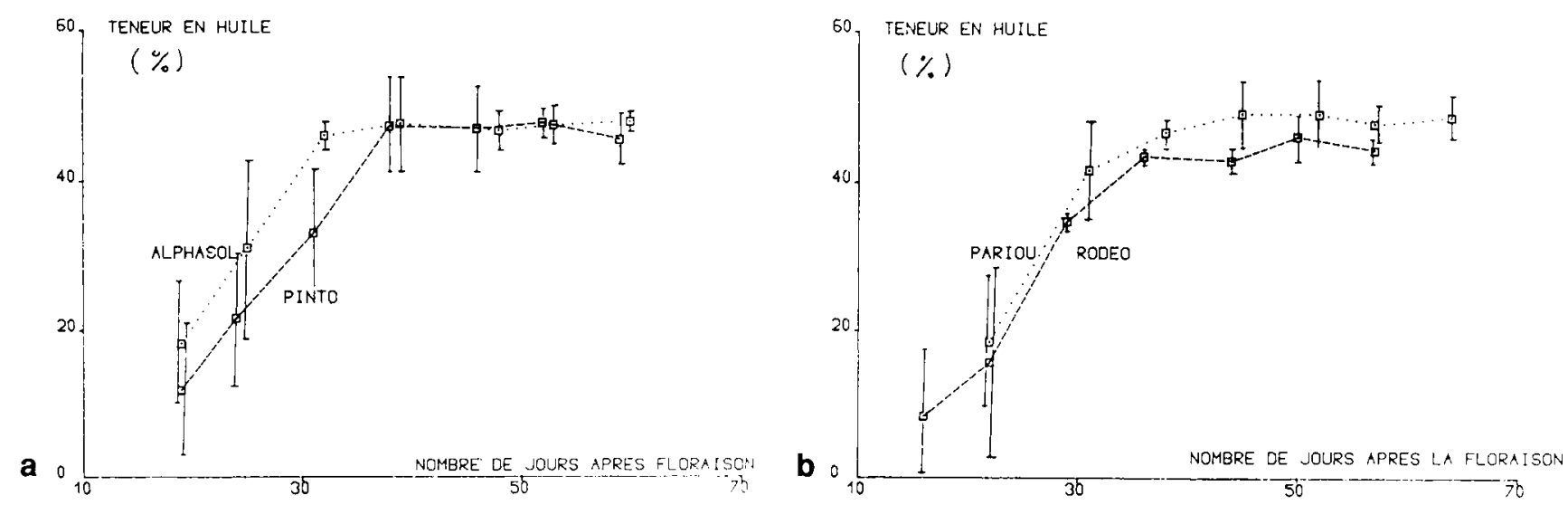

Fig. 2a et b. Evolution, au cours du temps, de la teneur en huile (intervalle pour un coefficient de sécurité de 95\%).

Tableau II. Valeurs des paramètres étudiés relatifs à la teneur en huile.

$\begin{array}{llll}\text { Teneur en huile } & \text { Nb.j. après } & \text { Humidité } & \text { Vitesse d'évolution } \\ 20 \text { j. après } & \text { floraison } & \text { au maximum } & \text { de la teneur en } \\ \text { floraison } & \text { pour max. } & \text { de teneur } & \text { huile en } \\ \text { en \% poids sec } & \text { teneur huile } & \text { en huile } & \text { point d'huile/jour }\end{array}$

Teneur en huile à la dernière observation en \% poids sec Moyenne groupes homogènes (Newmann-Keuls à 5\%)

\begin{tabular}{lrrrrrr}
\hline Viki & 18 & 41 & 33 & 2,91 & 52,10 & $\mathrm{a}$ \\
Véraflor & 10 & 49 & 35 & 2,41 & 51,81 & $\mathrm{a}$ \\
Vitaflor & 31 & 35 & 45 & 1,44 & 51,39 & $\mathrm{a}$ \\
Mirasol & 13 & 43 & 37 & 2,46 & 51,01 & $\mathrm{a}$ \\
Sémira & 26 & 38 & 40 & 1,64 & 50,76 & $\mathrm{a}$ \\
Sunking & 21 & 41 & 35 & 3,20 & 50,25 & $\mathrm{a}$ \\
IBH 166 & 26 & 42 & 34 & 2,81 & 49,08 & $\mathrm{a}$ \\
Pariou & 18 & 45 & 39 & 2,58 & 48,68 & $\mathrm{a}$ \\
Frankasol & 21 & 37 & 44 & 1,79 & 48,58 & $\mathrm{a}$ \\
IS 7111 & 9 & 39 & 50 & 2,33 & 48,13 & $\mathrm{a}$ \\
Alphasol & 18 & 32 & 45 & 1,24 & 47,80 & $\mathrm{ab}$ \\
Pinto & 12 & 38 & & 1,85 & 45,40 & $\mathrm{bc}$ \\
Rodéo & 8 & 36 & & 44,37 & $\mathrm{c}$ \\
\hline
\end{tabular}

${ }^{\star \star}$ : La vitesse d'évolution est calculée sur la période d'augmentation linéaire de la teneur en huile.

poids frais de la graine, incluant tous ses composants.

La teneur en eau des graines commence à diminuer dès le début des observations. En revanche, quelle que soit la variété, l'humidité du capitule ne varie pas pendant au moins 6 semaines après la floraison, sa teneur en eau se situant entre 70 et $80 \%$. Ensuite, elle décroît de façon différente suivant les variétés. En fonction du comportement pour le dessèchement des 2 organes, on peut établir 4 groupes de variétés:

Groupe I. Variétés présentant un décrochement dans la dessiccation des graines et un fort dessèchement du capitule (humidité inférieure à
$50 \%$ ) entre le 40 et le $50 \mathrm{e}$ jour après la floraison (Fig. 3a) : Alphasol, Pinto, Primasol, Vitaflor).

Groupe II. Variétés présentant une dessiccation très progressive des graines et un fort dessèchement du capitule 50 jours après la floraison (humidité inférieure à 50\% (Fig. 3b) : Frankasol, Maryflor, Mirasol, Cargisol, Sirio, Viki, Prestiflor, DKS 39, Sémira).

Groupe III. Variétés présentant une dessiccation progressive des graines et un faible et tardif dessèchement du capitule (humidité comprise entre 50 et $75 \%$ (Fig. 3c) : Isomax, IBH 166, Elia, Flamme, Pariou, Rodéo, Sunking, Véraflor). 

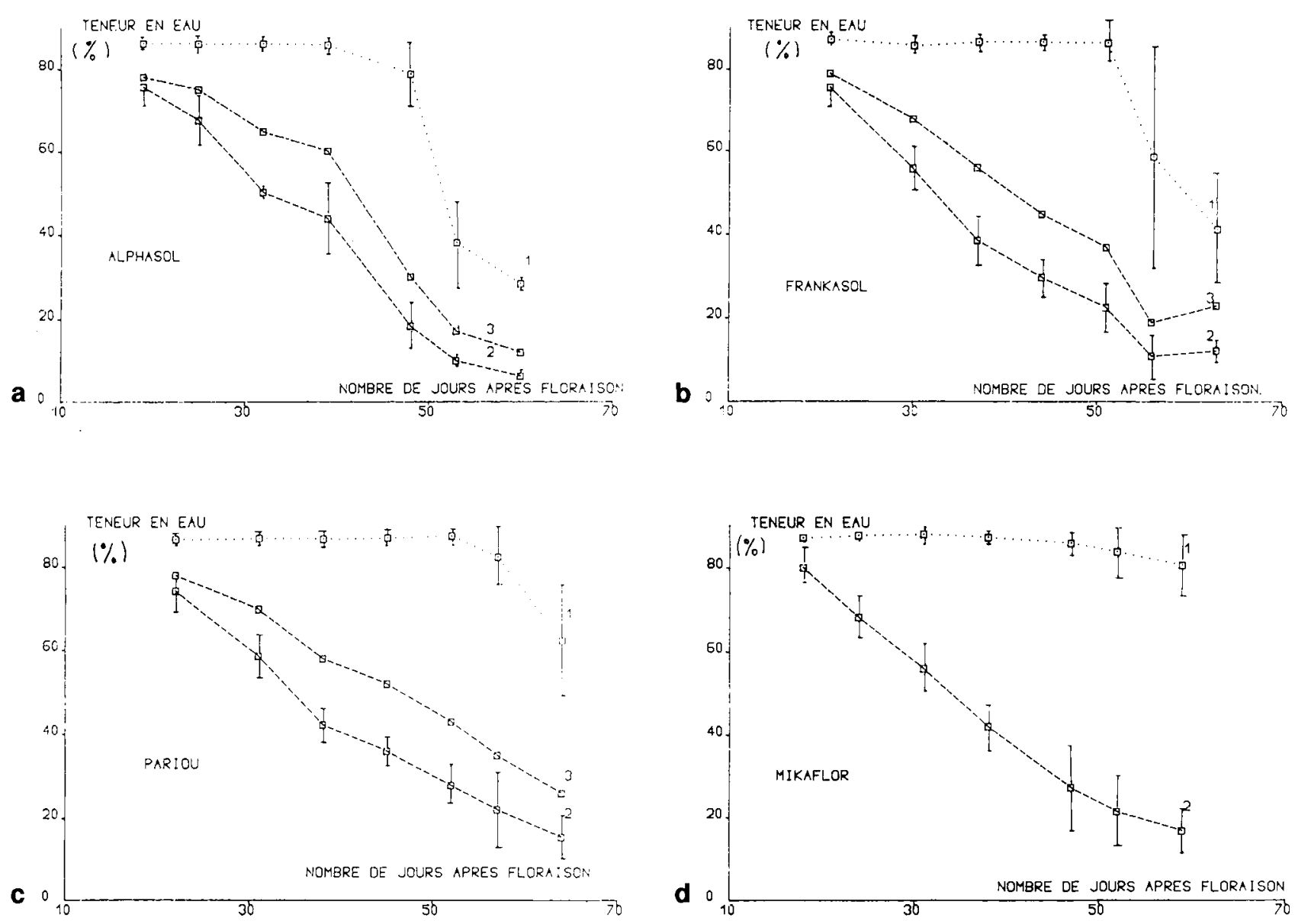

Fig. 3a, b, c et d. Evolution de l'humidité des capitules (1), des graines entières (2), et de la fraction non lipidique des graines (3) (intervalle de confiance pour un coefficient de sécurité de $95 \%$ ). a : groupe I, précoce avec décrochement de l'humidité des graines; b : groupe II, forte diminution de l'humidité du capitule; c : groupe III, faible diminution de l'humidité du capitule; $\mathbf{d}$ : groupe IV, pas de diminution de l'humidité du capitule.

Groupe IV. Variétés chez qui l'humidité du capitule ne diminue presque pas (Fig. 3d) : Cerflor, Mikaflor, Siou, IS7111.

On peut, sur les courbes, déterminer la date de diminution de la teneur en eau du capitule et la teneur en eau de la graine correspondante (Tableau III). La relation pourcentage d'humidité de la graine et date entre 3 et 10 semaines après floraison peut être ajustée à une régression linéaire hautement significative dans tous les cas. Les pentes des droites obtenues représentent les vitesses de dessiccation qui sont portées dans le Tableau III. Les variétés présentent des vitesses de dessèchement différentes. Ainsi, Alphasol perd 1,84 point d'eau par jour, Sémira 1,39. La floraison tardive de Sémira est associée à une vitesse de dessiccation faible, alors que la floraison précoce de Pinto est associée à une vitesse plus élevée, 1,81. Cependant, pour une même date de floraison, la vitesse de dessiccation peut être différente.

D'après le Tableau III, on peut voir que la variété la plus précoce à la floraison est Sunking, qui fleurit le 2 juillet, la plus tardive est Sémira qui fleurit le 13 juillet, soit une différence de 11 jours. Pour l'humidité au 2 septembre les variétés les plus précoces sont Alphasol $(6,21 \%)$ et Vitaflor $(6,64 \%)$, la plus tardive est Sémira $(26,62 \%)$, soit une différence de $20 \%$. II y a cependant une grande variabilité intragénotypes qui fait qu'une différence de 10 points n'est pas significative (test de Neuman-Keuls à $5 \%$ ).

Le nombre de jours après floraison pour lequel la teneur en eau du capitule diminue va de $\mathbf{4 0}$ jours, pour Alphasol et Primasol, à 57 jours, pour Véraflor et Elia. Pour certains génotypes, il n'y a pas de diminution. Ces valeurs correspondent à une humidité de la graine de $40 \%$ pour les 2 premières variétés et de $20 \%$ pour les 2 autres.

La régression (date de floraison) - (humidité au 2 septembre) est significative, cependant le coefficient de corrélation est faible $(r=0,42$, $23 \mathrm{ddl}$ ); ainsi, pour une même date de floraison, le 8 juillet, l'humidité varie de 7 à $20 \%$. La régression (vitesse de dessiccation) - (humidité au 2 septembre) est hautement significative $(r=0,71$, 
$23 \mathrm{ddl}$ ). L'humidité des graines à cette date est donc fonction de leur vitesse de dessèchement. La régression (nombre de jours après floraison pour la diminution en eau du capitule) - (humidité de la graine au 2 septembre) est hautement significative $(r=0,62,23 \mathrm{ddl})$. Cependant, chez Sémira et Frankasol, la teneur en eau du capitule diminue 50 jours après floraison alors qu'il y a 16 points d'humidité d'écart entre les 2 variétés. Entre la teneur en eau du capitule au 2 septembre et la teneur en eau des graines à la même date, on obtient une régression exponentielle très hautement significative $(r=0,96,23 \mathrm{ddl})$.

\section{Relation entre les différents paramètres}

Les régressions linéaires totales entre l'humidité au 2 septembre, la teneur en huile et le poids de 100 graines ne sont pas significatives.

On a les résultats suivants :

- huile-humidité : $r=0,30$ (11 ddl);
- huile-poids de 100 graines : $r=-0,14(8 \mathrm{ddl})$;

- poids de 100 graines-humidité $: r=0,10(8 \mathrm{ddl})$.

Le maximum de teneur en huile est atteint, en moyenne, 8 jours avant le maximum de poids sec.

\section{Discussion}

\section{Evolution du poids sec de 100 graines}

Pour l'évolution du poids sec de 100 graines, les variétés diffèrent en durée mais surtout en vitesse. On doit rappeler que les mesures ont été effectuées sur un échantillon et non sur le capitule entier. Les différences pourraient être liées au nombre de graines par capitule à alimenter et aussi à des différences de quantité d'assimilats. Rollier et al. (1976) ont montré, sur le tournesol, que le poids de 1000 graines s'accroît régulièrement, au cours de la phase de formation

Tableau III. Valeur des différents paramètres relatifs à l'humidité des graines et des capitules.

\begin{tabular}{|c|c|c|c|c|c|c|c|}
\hline & $\begin{array}{l}\text { Date de } \\
\text { floraison } \\
\text { en juillet }\end{array}$ & $\begin{array}{l}\text { Humidit } \\
\text { au } 2 / 09 \\
\text { Moyenr } \\
\text { groupe } \\
\text { N-K à } 5\end{array}$ & $\begin{array}{l}\text { s graines } \\
n \% \\
\text { nogènes }\end{array}$ & $\begin{array}{l}\text { Vitesse de } \\
\text { dessiccation } \\
\text { cle la graine } \\
\text { en \% par jour } \\
\text { entre } 3 \text { et } 10 \\
\text { semaines après } \\
\text { la floraison }\end{array}$ & $\begin{array}{l}\text { Nombre de jours } \\
\text { après floraison } \\
\text { pour diminution } \\
\text { en eau capitule }\end{array}$ & $\begin{array}{l}\text { Humidité de la } \\
\text { graine en \% } \\
\text { pour diminution } \\
\text { graines/capitule }\end{array}$ & $\begin{array}{l}\text { Groupe } \\
\text { pour la } \\
\text { dessication }\end{array}$ \\
\hline Alphasol & 4 & 6,21 & $a$ & 1,84 & 40 & 40 & 1 \\
\hline Vitaflor & 8 & 6,64 & $a$ & 1,76 & 43 & 35 & 1 \\
\hline Isomax & 8 & 9,27 & $a b$ & 1,64 & 49 & 20 & 3 \\
\hline Pinto & 5 & 9,46 & $a b$ & 1,81 & 46 & 35 & 1 \\
\hline IBH 166 & 9 & 9,79 & $a b$ & 1,66 & 48 & 23 & 3 \\
\hline Primasol & 4 & 10,10 & $a b$ & 1,76 & 40 & 40 & 1 \\
\hline Sunking & 2 & 10,58 & $a b$ & 1,40 & 55 & 28 & 3 \\
\hline Frankasol & 8 & 10,97 & $a b$ & 1,56 & 50 & 25 & 2 \\
\hline Rodéo & 7 & 11,87 & $a b c$ & 1,70 & 50 & 30 & 3 \\
\hline Prestiflor & 8 & 12,04 & $a b c$ & 1,62 & 50 & 25 & 2 \\
\hline DKS 39 & 9 & 13,06 & $a b c$ & 1,66 & 48 & 25 & 2 \\
\hline Mirasol & 8 & 13,11 & $a b c$ & 1,63 & 50 & 29 & 2 \\
\hline Viki & 10 & 13,94 & $a b c$ & 1,53 & 47 & 29 & 2 \\
\hline Cargisol & 7 & 14,26 & $a b c$ & 1,63 & 50 & 30 & 2 \\
\hline Sirio & 9 & 14,26 & $a b c$ & 1,63 & 50 & 30 & 2 \\
\hline Cerflor & 6 & 15,26 & $a b c$ & 1,66 & * & 1 & 4 \\
\hline Maryflor & 9 & 15,82 & $a b c$ & 1,61 & 50 & 29 & 2 \\
\hline Siou & 7 & 16,44 & $a b c$ & 1,44 & * & I & 4 \\
\hline Mikaflor & 5 & 16,53 & $a b c$ & 1,60 & * & 1 & 4 \\
\hline Véraflor & 9 & 17,22 & bcd & 1,53 & 57 & 20 & 3 \\
\hline IS 7111 & 4 & 19,26 & bcd & 1,61 & $\star$ & 1 & 4 \\
\hline Elia & 8 & 19,34 & bcd & 1,57 & 57 & 21 & 3 \\
\hline Pariou & 7 & 22,12 & bcd & 1,39 & 52 & 30 & 3 \\
\hline Flamme & 10 & 22,93 & $\mathrm{~cd}$ & 1,50 & 55 & 23 & 3 \\
\hline Sémira & 13 & 26,62 & $d$ & 1,39 & 50 & 30 & 2 \\
\hline
\end{tabular}

* Pas de diminution. 
des akènes, jusqu'à la maturité. Cependant, Rodrigues Pereira (1978), Browne (1978) et Ortegon Morales (1980) ont montré, comme dans notre étude, que la matière sèche de la graine atteint un maximum entre 30 et 50 jours après la floraison. D'après Anderson (1975) et Unger et Thompson (1982), le mode d'accumulation de la matière sèche varie en fonction de la date de semis et des conditions environnementales. Ce schéma d'évolution de la matière sèche, qui présente un palier en fin de cycle, s'observe aussi chez le soja (Crookston et Hill, 1978; Swank et al., 1987) et le maïs (Verdier, 1983; Bloc et al., 1983).

Nous avons observé, chez le tournesol, que la taille de la graine est en relation avec sa vitesse de croissance. Chez le soja, la taille de la graine est déterminée par la vitesse et la durée de remplissage, les 2 étant sous contrôle génétique (Egli, 1975; Egli et al., 1981; Egli et al., 1984), bien qu'ils puissent varier en fonction des conditions du milieu (Egli et Wardlaw, 1980). Les mêmes résultats ont été observés chez le blé (Bruckner et Frohberg, 1987). Cependant, dans certains cas, seule la vitesse de croissance détermine la taille de la graine : exemples du soja (Egli et al., 1981) et du riz (Jones et al., 1979).

La taille de la graine est associée au taux d'accumulation de la matière sèche et au nombre de cellules de l'embryon ou de l'albumen chez un certain nombre d'espèces. Brocklehurst (1977) a montré, chez deux cultivars de blé, que le taux de croissance de la graine et sa taille finale sont liés au nombre de cellules de l'albumen. De la même façon, Reddy et Daynard (1983) ont montré que le nombre de cellules de l'albumen, le nombre de granules d'amidon, le taux de croissance et la taille de la graine sont positivement corrélés chez 3 hybrides de maïs. Les travaux de Jones et al. (1985) sur le maïs vont dans le même sens. Chez le pois, il existe une relation positive entre le nombre de cellules des cotylédons et la matière sèche finale de la graine (Davies, 1975). Chez le soja, Egli et al. (1981) ont montré que les différences génétiques dans le taux de croissance de la graine sont liées au nombre de cellules des cotylédons.

Chez le tournesol, aucune étude de ce genre n'a été menée jusqu'à présent. Cependant, il est vraisemblable que 2 systèmes différents soient à considérer : la coque et l'amande.

\section{Teneur en huile}

Nous avons trouvé une évolution presque sigmoïdale de la teneur en huile qui est en accord avec les résultats des autres auteurs (Anderson, 1975; Simpson et Radford, 1976; Robertson et al., 1978; Rodrigues Pereira, 1978; Ortegon Moralès, 1980; Unger et Thompson, 1982). Ainsi, la quantité totale d'huile est formée, suivant les variétés, entre 30 et 50 jours après floraison, ce qui correspond à une humidité de la graine de 33 à 50\%. Les résultats d'Anderson (1975) ont montré que la teneur maximale est atteinte pour des durées après floraison différentes suivant la date de semis; Simpson et Radford (1976) ont montré que ce palier est atteint entre. 35 et 50 jours après la floraison suivant la date de semis; pour Ortegon Morales (1980), il est atteint vers 30 jours après la floraison et pour une humidité de la graine de $24 \%$. Grewal et al. (1978) ont montré qu'il y a une synthèse importante d'huile entre 10 et 30 jours après fécondation, avec un pic entre 20 et 30 jours. D'après Sukhija et al. (1980) et Monga et al. (1983), le pic se situe entre 10 et 20 jours. Ces derniers auteurs ont étudié les graines des 3 rangs extérieurs alors que nous avons étudié les rangs médians du capitule. Ce type de biosynthèse des lipides qui présente un palier a été retrouvé chez le colza (Norton et Harris, 1975) et le carthame (Ichihara et Noda, 1980).

\section{Humidité}

Chez le tournesol, les auteurs trouvent des modes de dessèchement de la graine différents. Ainsi, Anderson (1975) et Rollier et al. (1976) ont montré que la dessiccation du grain est régulière au cours du temps. Cependant, Rodrigues Peireira (1978) a trouvé, en conditions contrôlées, que la vitesse de dessèchement est d'abord lente pendant les 2 premières semaines après la fécondation, puis l'humidité se met à décroître plus rapidement. A partir de $30 \%$ d'eau, soit 52 à 70 jours après floraison, suivant les conditions, il y a une diminution beaucoup plus importante, jusqu'à $10 \%$ d'eau. Un comportement non régulier n'a été trouvé, dans notre étude, que pour le groupe $\mathrm{I}$, où il y a un décrochement dans l'humidité des graines entre le $40^{\circ}$ et 50 e jour après la floraison. Chez le triticale (Bishnoi, 1974) et le blé (Sofield et al., 1977), la vitesse de dessiccation du grain augmente après la maturité physiologique.

En conditions non irriguées, Rollier et al. (1976) ont trouvé des diminutions de teneur en eau de 0,88 point par jour en 1973, de 1,45 en 1974 et de 1,25 en 1975, sans qu'il y ait de différences significatives entre les variétés. C'est un exemple de la forte influence des conditions climatiques sur la vitesse de dessication. Les conditions très sèches de 1987 ont conduit à des vitesses de dessèchement élevées (entre 1,84 et 1,39). 
En accord avec nos résultats, Anderson (1975) et Rollier et al. (1976) ont mis en évidence que la teneur en eau du capitule ne variait pas pendant un certain temps et diminuait ensuite. La diminution se situe vers 40 jours après floraison pour la variété étudiée par Anderson. Pour les variétés que nous avons étudiées, elle a lieu entre 40 et 57 jours après la floraison. Rollier et al. (1976) ont montré que la teneur en eau du capitule commence à diminuer lorsque la graine atteint $18 \%$ d'eau chez le cv. Pérédovick et $10 \%$ chez les autres variétés.

Ainsi, au travers des variétés, il ne semble pas exister de relation entre les dessèchements du capitule et des graines, dans la mesure où ces dernières sèchent à la même vitesse, quel que soit l'état physiologique du capitule, et que leur teneur en eau peut diminuer sans variation de la teneur en eau du capitule. Cependant, les variétés les plus précoces au 2 septembre sont celles qui possèdent le capitule le moins riche en eau.

Nous avons voulu savoir si la diminution d'humidité que l'on observe était une dessiccation réelle ou était due à l'augmentation de la teneur en huile sans perte d'eau. Nos résultats montrent qu'il y a une perte d'eau réelle dès le $20^{\circ}$ jour après la floraison.

Chez le maïs, Purdy et Crane (1967) ont montré que la vitesse de dessèchement du grain est en relation avec l'épaisseur du péricarpe. Chez le tournesol, la coque, qui représente 10 à $40 \%$ du poids $\mathrm{sec}$, doit avoir un rôle non négligeable dans la déshydratation de l'akène. II serait intéressant de déterminer s'il y a une relation entre son épaisseur et la vitesse de dessiccation.

\section{Maturité physiologique}

Pour Anderson (1975), la maturité physiologique est atteinte lorsque le poids des graines et la teneur en huile sont maximaux. Dans notre étude, le poids sec maximal du grain est atteint environ une semaine après le maximum de teneur en huile. D'après Anderson (1975) et Ortegon Morales (1980), les 2 sont atteints en même temps. II est à noter que nous avons trouvé une variabilité beaucoup plus importante pour le poids de 100 graines que pour la teneur en huile. Pour différents génotypes et différentes conditions d'expérimentation, les auteurs ont trouvé que la date de maturité physiologique et la teneur en eau correspondante variaient : 31 jours après la floraison avec une teneur en eau de $30 \%$ pour Vniimk 6540 (Browne, 1978); 35 jours après floraison avec $36 \%$ d'eau pour Sungro (Roberston et al., 1978); respectivement 32\% et $34 \%$ pour Sunfola $68-2$ et Hysun 30 (Goyne et al., 1979).
Le maximum de poids sec du grain est atteint à 40\% d'humidité chez le blé (Scott et al., 1957), à $23-30 \%$ chez le sorgho (Kersing et al., 1961), à $30-37 \%$ chez le maïs (Daynard, 1972) et à 54$62 \%$ chez le soja (Tekrony et al., 1979). Cependant, la teneur en eau du grain n'est pas un critère très fiable pour déterminer la maturité physiologique, car cette dernière est fonction des conditions climatiques. C'est pourquoi des critères visuels ont été recherchés. Ainsi, chez le maïs (Daynard et Duncan, 1969) et le sorgho (Eastin et al., 1973), il se forme à la maturité physiologique une zone de subérisation ou black layer. Chez le soja, ce stade est atteint lorsque la graine est complètement jaune (Crookston et Hill, 1978; Tekrony et al., 1979). Chez l'avoine (Lee et al., 1979) et l'orge (Copeland et Crookston, 1985), la maturité physiologique correspond au brunissement des glumes. Chez le tournesol, ce stade se repère par une couleur jaune du capitule et brune des bractées. Chez le maïs, la formation du black layer stoppe les migrations d'assimilats vers les graines. Chez le tournesol, il serait intéressant de préciser les processus mis en jeu.

\section{Conclusion}

Cette étude décrit les phénomènes qui interviennent lors de la maturation des akènes de tournesol : augmentation de la matière sèche et de la teneur en huile des graines et diminution de teneur en eau des graines et du capitule. Nous n'avons que des hypothèses pour expliquer les différences variétales. De nombreux travaux restent nécessaires. II serait intéressant d'étudier entre autres le rôle possible de la coque dans ce processus.

\section{Remerciements}

Nous tenons à remercier la coopérative Dom-Agri pour sa coopération dans le suivi de cette expérimentation.

\section{Références}

Anderson W.K. (1975) Maturation of sunflower. Aust. J. Exp. Agric. Anim. Husb. 15, 833-838

Bloc D., Gay J.P. \& Gouet J.P. (1983) Evolution de la teneur en eau et du poids de 1000 grains pendant la maturation du maïs. Colloque Physiologie du maïs, Royan (France), 17 mars 1983. INRA

Bishnoi V.R. (1974) Physiological maturity of seeds in Triticale hexaploid L. Crop Sci. 14, 819-821

Brocklehurst P.A. (1977) Factor controlling grain weight in wheat. Nature 266, 348-349 
Browne C.L. (1978) Identification of physiological maturity in sunflower (Helianthus annuus). Aust. $J$. Exp. Agric. Anim. Husb. 18, 282-286

Bruckner P.L. \& Frohberg R.C. (1987) Rate and duration of grain fill in spring wheat. Crop Sci. 27, 451-455

Copeland P.J. \& Crookston R.K. (1985) Visible indicators of physiological maturity in barley. Crop Sci. 25, 843-847

Crookston R.K. \& Hill D.S. (1978) A visual indicator of the physiological maturity of soybean seed. Crop Sci. $18,867-870$

Davies D.R. (1975) Studies of seed development in Pisum sativum. I. Seed size. Planta 124, 297-302

Daynard T.B. \& Duncan W.G. (1969) The black layer and grain maturity in corn Crop Sci. 9, 473-476

Daynard, T.B. (1972) Relationships among black layer formation, grain moisture percentage, and heat unit accumulation in corn. Agron. J. 64, 716-719

Eastin J.D., Hultquist J.H. \& Sullivan C.Y. (1973) Physiological maturity in grain sorghum. Crop Sci. 9, 473476

Egli D.B. (1975) Rate of accumulation of dry weight in seed of soybeans and its relationship to yield. Can. J. Plant Sci. 55, 215-219

Egli D.B. \& Wardlaw I.F. (1980) Temperature response of seed growth caracteristics of soybeans. Agron. J. 72, 560-564

Egli D.B., Fraser J., Legett J.E. \& Poneleit C.G. (1981) Control of seed growth in soya beans (Glycine max (L.) Merril). Ann. Bot. 48, 176-181

Egli D.B., ORF J.H. \& Pfeiffer T.W. (1984) Genotypic variation for duration of seedfill in soybean. Crop Sci. 24, 587-592

Goyne P.J., Simpson B.W., Woodruff D.R. \& Churchett J.D. (1979) Environmental influence on sunflower achene growth, oil content and oil quality. Aust. J. Exp. Agric. Anim. Husb. 19, 82-88

Gradlung H. \& Zimmerman D.C. (1975) Effect of drying conditions on oil content of sunflower seeds as determinated by wide-line nuclear magnetic resonance. $N$. Dakota Acad. Sci. Proc. 27, 2, 128-132

Grewal S.S., Sukhija P.S. \& Bathia I.S. (1978) Polar lipid composition in sunflower (Helianthus annuus) seed development. Biochem. Physiol. Pflanzen 173, $11-16$

Harington J.F. (1972) Seed storage and longevity. In: Seed Biology. Vol. 3, (T.T. Kozlowski, ed.) Academic Press, New York

Ichihara K.I. \& Noda M. (1980) Fatty acid composition and lipid synthesis in developping safflower seeds. Phytochemistry 19, 49-54

Jones D.B., Petterson M.C. \& Geng S. (1979) Association between grain filling rate and yield components in rice. Crop Sci. 19, 641-644

Jones R.J., Roessler J. \& Ouattar S. (1985) Thermal environment during endosperm cell division in maize : effects on number of endosperm cells and starch granules. Crop Sci. 25, 830-834

Kersting J.F., Stickler F.C. \& Pauli A.W. (1961) Grain sorghum caryopsis development. 1-Changes in dry weigth, moisture percentage and viability. Agron. J. 53 , 36-38

Lee H.J., McKee G.W. \& Knievel D.D. (1979) Determination of physiological maturity in oat. Agron. J. 71, 931-935

Monga P.K., Munshi S.K. \& Sukhija P.S. (1983) Lipid biosynthesis in the developping sunflower (Helianthus annuus L.) seeds. Plant Sci. Lett. 31, 311-321

Northon G. \& Harris F.J. (1975) Compositional changes in developping rape seeds (Brassica napus L.). Planta 123, 163-174

Ortegon Morales A.S. (1980) Etapa de la madurez fisiologica del girasol (Helianthus annuus). Agric. Tec. Mex. 6, 1, 29-33

Purdy J.L. \& Crane P.L. (1967) Influence of pericarp on differencial drying rate in mature corn (Zea mays L.). Crop Sci. 7, 379-381

Reddy V.M. \& Daynard T.B. (1983) Endosperm characteristics associated with rate of grain filling and kernel size in corn. Maydica 28, 339-355

Robertson J.A., Chapman G.W., Jr. \& Wilson R.L., Jr. (1978) Relation of days after flowering to chemical composition and physiological maturity of sunflower seed. J. Am. Oil Chem. Soc. 55, 266-269

Robinson R.G. (1983) Maturation of sunflower and sector sampling of heads of monitor maturation. Field Crops Res. 7, 31-39

Rodrigues Pereira A.S. (1978) Influence of temperature on seed growth and seed ripening in sunflower. 8th Int. Sunflower Conference Minneapolis, USA. 199-211

Rollier M., Bughart P. \& Chanet M. (1976) Evolution des caractéristiques de la graine et du capitule au cours de la maturation. 7e Conférence Internationale sur le Tournesol, Krasnodar, URSS. 276-284

Scott G.E., Heyne E.G. \& Finney K.F. (1957) Development of the hard red winter wheat kernel in relation to yield, kernel weight, moisture content and milling and baking quality. Agron. J. 49, 509-513

Simpson B.W. \& Radford B.J. (1976) Levels of moisture, oil, nitrogen and fatty acid in the maturing seed of sunflower (Helianthus annuus). Queensl. J. Agric. Anim. Sci. 33, 2, 189-201

Sofield I., Wardlaw I.F., Evans L.T. \& Zee S.Y. (1977) Nitrogen, phosphorus and water contents during grain development and maturation in wheat. Aust. J. Plant Physiol. 4, 799-810

Sukhija P.S., Borthakur A. \& Bathia I. (1980) Effect of irrigation on lipid biosynthesis in maturing sunflower seeds. J. Sci. Food Agric. 31, 225-228

Swank J.C., Egli D.B. \& Pfeiffer T.W. (1987) Seed growth characteristics of soybean genotypes differing in duration of seed fill. Crop Sci. 27, 85-89

Tekrony D.M., Egli D.B., Balles J., Pfeiffer T. \& Fellows R.J. (1979) Physiological maturity in soybean. Agron. J. $71,771-775$

Unger P.W. \& Thompson T.E. (1982) Planting date effect on sunflower head and seed development. Agron. J. 74, 389-395

Verdier P. (1983) Approche écophysiologique de la sélection du maïs précoce. Thèse de doctorat, Université de Clermont-II 